\title{
Compatibility between environment-induced decoherence and the modal-Hamiltonian interpretation of quantum mechanics
}

\author{
Olimpia Lombardi - Sebastian Fortin - Mario Castagnino - Juan Sebastián Ardenghi \\ CONICET - Faculty of Exact and Natural Sciences, University of Buenos Aires
}

\begin{abstract}
Given the impressive success of environment-induced decoherence (EID), nowadays no interpretation of quantum mechanics can ignore its results. The modal-Hamiltonian interpretation (MHI) has proved to be effective for solving several interpretative problems but, since its actualization rule applies to closed systems, it seems to stand at odds of EID. The purpose of this paper is to show that this is not the case: the states einselected by the interaction with the environment according to EID (the elements of the "pointer basis") are the eigenvectors of an actualvalued observable belonging to the preferred context selected by the MHI.
\end{abstract}




\title{
Compatibility between environment-induced decoherence and the modal-Hamiltonian interpretation of quantum mechanics
}

\author{
Olimpia Lombardi - Sebastian Fortin - Mario Castagnino - Juan Sebastián Ardenghi \\ CONICET - Faculty of Exact and Natural Sciences, University of Buenos Aires
}

\section{Introduction}

The modal interpretations of quantum mechanics are realist, non-collapse interpretations, according to which the quantum state describes the possible properties of the system, rather than the actually possessed properties (for overview and references, see Dieks and Vermaas 1998). Each modal version proposes a specific interpretative rule of actual-value ascription, in general with the aim of offering and adequate answer to the quantum measurement problem. Some of them work very well in the account of ideal measurements, but face severe problems in the non-ideal case. It is at this point that environment-induced decoherence (EID) entered the discussion: some authors suggested that, since measuring apparatuses are never isolated from their environments, decoherence provides an answer to the non-ideal-measurement challenges.

Recently a new interpretation belonging to the modal family has been proposed: according to the modal-Hamiltonian interpretation (MHI), the Hamiltonian of the closed system defines the preferred context -the set of the actual-valued observables-. The MHI has proved to be effective for solving the measurement problem, both in its ideal and its non-ideal versions, and has been applied to many well-known physical situations (free particle with spin, harmonic oscillator, hydrogen atom, Zeeman effect, fine structure, Born-Oppenheimer approximation) leading to results compatible with experimental evidence.

Since immune to the non-ideal-measurement challenges, the MHI has no need of decoherence for giving an adequate account of quantum measurement. Nevertheless, to the extent that the preferred context is defined by the Hamiltonian of the system -conceived as a closed system with no external interaction-, the MHI seems to be incompatible with the EID approach, which relies on the interaction between the measurement apparatus -an open system- and its environment. In this paper we shall argue that the conflict is merely apparent: in the measurement situation, the preferred context defined by the MHI agrees with the pointer basis selected by EID. 


\section{Modal Interpretations and Decoherence}

In the Kochen-Dieks (K-D) modal interpretation (Kochen 1985; Dieks 1988), the biorthogonal (Schmidt) decomposition of the pure quantum state of the system picks out the actual-valued observables. In the Vermaas-Dieks (V-D) version (Vermaas and Dieks 1995), a generalization of the K-D interpretation to mixed states, the actual-valued properties are defined by the spectral resolution of the system's reduced state, obtained by partial tracing. Both versions seem to be designed to give an answer to the ideal (perfect and non-disturbing) von Neumann measurement, where the interaction between the measured system $S$ and the measuring apparatus $M$ establishes a perfect correlation between the eigenstates $\left|o_{i}\right\rangle$ of an observable $O$ of $S$ and the eigenstates $\left|p_{i}\right\rangle$ of a pointer observable $P$ of $M$,

$$
\left|\psi_{0}\right\rangle=\sum_{i} c_{i}\left|o_{i}\right\rangle \otimes\left|p_{0}\right\rangle \rightarrow|\psi\rangle=\sum_{i} c_{i}\left|o_{i}\right\rangle \otimes\left|p_{i}\right\rangle
$$

In fact, in the ideal situation the expansion of the correlated state in terms of the eigenvectors of $O$ and $P$ is a biorthogonal decomposition, and

$$
\rho_{S}=\operatorname{Tr}_{M}(|\psi\rangle\langle\psi|)=\sum_{i}\left|c_{i}\right|^{2}\left|o_{i}\right\rangle\left\langle\left. o_{i}\left|\quad \rho_{M}=\operatorname{Tr}_{A}(|\psi\rangle\langle\psi|)=\sum_{i}\right| c_{i}\right|^{2} \mid p_{i}\right\rangle\left\langle p_{i}\right|
$$

As a consequence, according to the K-D and the V-D rules of actual value-ascription, both the observable $O$ and the pointer $P$ are actual-valued observables (see also the Copenhagen Variant by van Fraassen 1991).

However, the von Neumann model is a never attainable idealization: a real measurement always involves small but non-zero cross-terms due to imperfect correlations (first kind measurement):

$$
|\psi\rangle=\sum_{i j} d_{i j}\left|o_{i}\right\rangle \otimes\left|p_{j}\right\rangle=\sum_{i} d_{i}\left|o_{i}^{*}\right\rangle \otimes\left|p_{i}\right\rangle \text { with } d_{i j} \neq 0 \text { for } i \neq j,\left\langle o_{i}^{*} \mid o_{j}^{*}\right\rangle \neq \delta_{i j}
$$

Furthermore, it can be expected that a real measurement introduces a disturbance onto the measured system (second kind measurement):

$$
|\psi\rangle=\sum_{i} d_{i}\left|o_{i}^{*}\right\rangle \otimes\left|p_{i}\right\rangle=\sum_{i j} d_{i j}\left|o_{i}\right\rangle \otimes\left|p_{j}\right\rangle \text { with }\left\langle o_{i}^{*} \mid o_{j}^{*}\right\rangle \neq \delta_{i j}, d_{i j} \neq 0 \text { for } i \neq j
$$

As noticed by Albert and Loewer (1990, 1993), Albert (1992), Elby (1993) and Ruetsche (1996), among others, in both kinds of measurement the K-D and the V-D interpretations lead to results that disagree with those obtained in the orthodox collapse interpretation. Moreover, whereas in the case of an imperfect measurement it can be expected that the $d_{i j} \neq 0$, with $i \neq j$, be small, in the disturbing case they need not be small and, as a consequence, the disagreement might be unacceptable. 
It is at this point that EID has been appealed to in order to protect modal interpretations from the non-ideal-measurement challenges (see Healey 1989, 1995; Dieks 1994a, 1994b; Bacciagaluppi and Hemmo 1996; Monton 1999). In fact, the measuring apparatus is in interaction with a very large environment $E$, whose states associated with the different pointer states $\left|p_{i}\right\rangle$ can be expressed as $\left|e_{i}(t)\right\rangle$. The state of the whole system $S+M+E$ is, then,

$$
|\psi\rangle=\sum_{i} c_{i}\left|o_{i}^{*}\right\rangle \otimes\left|p_{i}\right\rangle \otimes\left|e_{i}(t)\right\rangle
$$

The reduced density operator of the apparatus is obtained by tracing over the degrees of freedom of $S$ and $E$,

$$
\rho_{M}(t)=\operatorname{Tr}_{S E}(|\psi(t)\rangle\langle\psi(t)|)=\sum_{i j} c_{i} c_{j}^{*}\left|p_{i}\right\rangle\left\langle p_{j}\right|\left\langle o_{j}^{*} \mid o_{i}^{*}\right\rangle\left\langle e_{j}(t) \mid e_{i}(t)\right\rangle
$$

where the factor $\left\langle e_{j}(t) \mid e_{i}(t)\right\rangle$ determines the size of the off-diagonal terms at each time. Many models for the interaction between $M$ and $E$ show that, when the environment is composed of a large number of subsystems, the states $\left|e_{i}(t)\right\rangle$ of the environment rapidly approach orthogonality and, as a consequence, $\rho_{M}(t)$ rapidly becomes approximately diagonal in the basis $\left\{\left|p_{i}\right\rangle\right\}$. In other words, after the decoherence time the off-diagonal elements of $\rho_{M}(t)$ are small independently of the original disturbance (for a full argument, see Bacciagaluppi and Hemmo 1996; for difficulties in continuous models, see Bacciagaluppi 2000).

\section{The EID Pointer Basis}

In his first papers on decoherence, Zurek $(1981,1982)$ studied physical models where the reduced density matrix ends up being diagonal in the eigenvectors of an observable $P$, which commutes with the Hamiltonian $H_{M E}^{\text {int }}$ describing the apparatus-environment interaction. According to Zurek, this property is what makes $P$ to be the pointer observable: since $P$ is a constant of motion of $H_{M E}^{\text {int }}$, when the apparatus is in one of its eigenstates, the interaction with the environment will leave it unperturbed: "The form of the interaction Hamiltonian between the apparatus and its environment is sufficient to determine which observable of the measured quantum system can be considered «recorded» by the apparatus. The basis that contains that record the pointer basis of the apparatus-consists of the eigenvectors of the operator which commutes with the apparatusenvironment interaction Hamiltonian" (Zurek 1981, 1516). Since those first works, the condition

$\left[P, H_{M E}^{\text {int }}\right]=0$ has usually be considered as the definition of the pointer basis or of the pointer $P$ of the apparatus. For instance, Elby $(1994,363)$ explains: "Let $P$ ' denote an arbitrary apparatus observable that doesn't commute with the pointer reading $P$. Using 'toy' examples, along with general considerations, Zurek argues that $H_{M E}^{\text {int }}$ commutes with $P$, but does not commute with any 
$P^{\prime}$. In rough terms, the interaction between the apparatus and its environment picks out the pointer-reading basis". More recently, Schlosshauer (2004, 1278-79) claims: "One can then find a sufficient criterion for dynamically stable pointer states that preserve the system-apparatus correlations in spite of the interaction of the apparatus with the environment by requiring all pointer state projection operators $P_{n}=\left|p_{n}\right\rangle\left\langle p_{n}\right|$ to commute with the apparatus-environment Hamiltonian $H_{M E}^{\text {int }} \cdot "$

In the 90's, Zurek stressed that the original definition of the pointer basis was a simplification: when the system's dynamics is relevant, the einselection of the preferred basis is more complicated. Zurek introduced the "predictability sieve" criterion (Zurek 1993, Zurek, Habib and Paz 1993) as a systematic strategy to identify the preferred basis in generic situations. The criterion relies on the fact that the preferred states are, by definition, those less affected by the interaction with the environment. On the basis of the application of this criterion, three different regimes for the selection of the preferred basis can be distinguished (Paz and Zurek 1999, 2002; see also Zurek 2003):

The first regime is the quantum measurement situation, where the self-Hamiltonian of the system can be neglected and the evolution is completely dominated by the interaction Hamiltonian. In such a case, the preferred states are directly the eigenstates of the interaction Hamiltonian (Zurek 1981).

The second regime is the more realistic and complex situation, where neither the selfHamiltonian of the system nor the interaction with the environment are clearly dominant, but both induce non-trivial evolution. In this case, the preferred basis arises from the interplay between self-evolution and interaction; quantum Brownian motion belongs to this case (Paz 1994).

The third regime corresponds to the situation where the dynamics is dominated by the system's self-Hamiltonian. In this case, the preferred states are simply the eigenstates of this selfHamiltonian (Paz and Zurek 1999).

\section{The Modal-Hamiltonian Interpretation}

The non-ideal-measurement criticisms affect those modal interpretations whose rules of actual-value ascription depend on the state of the system. On the contrary, the MHI rule only depends on the system's Hamiltonian. Let us recall the main postulates of this intepretation (Lombardi and Castagnino 2008; see also Castagnino and Lombardi 2008; Ardenghi, Castagnino and Lombardi 2009; Lombardi, Castagnino and Ardenghi 2010) 
A quantum system $S$ is a pair $(\mathcal{O}, H)$ such that (i) $\mathcal{O}$ is a space of self-adjoint operators on a Hilbert space $\mathcal{H}$, representing the observables of the system, (ii) $H \in \mathcal{O}$ is the time-independent Hamiltonian of the system, and (iii) if $\rho_{0} \in \mathcal{O}^{\prime}$ (where $\mathcal{O}^{\prime}$ is the dual space of $\mathcal{O}$ ) is the initial state of $S, \rho_{0}$ evolves according to the Schrödinger equation in its von Neumann version.

A quantum system $S:(\mathcal{O}, H)$ with initial state $\rho_{0} \in \mathcal{O}^{\prime}$ is composite when it can be partitioned into two quantum systems $S^{1}:\left(\mathcal{O}^{1}, H^{1}\right)$ and $S^{2}:\left(\mathcal{O}^{2}, H^{2}\right)$ such that (i) $\mathcal{O}=\mathcal{O}^{1} \otimes \mathcal{O}^{2}$, and (ii) $H=H^{1} \otimes I^{2}+I^{1} \otimes H^{2} \in \mathcal{O}$ (where $I^{1}$ and $I^{2}$ are the identity operators in the corresponding tensor product spaces). In this case, the initial states of $S^{1}$ and $S^{2}$ are obtained as the partial traces $\rho_{0}^{1}=\operatorname{Tr}_{(2)} \rho_{0}$ and $\rho_{0}^{2}=\operatorname{Tr}_{(1)} \rho_{0}$, and we say that $S^{1}$ and $S^{2}$ are subsystems of the composite system $S$. If the quantum system is not composite, we call it elemental.

Actualization rule: given an elemental quantum system $S:(\mathcal{O}, H)$, the preferred context consists of $H$ and the observables commuting with $H$ and having, at least, the same symmetries -degeneracies- as $H$.

According to the MHI, a quantum measurement is a three-stage process. In the first stage, the system $S$ to be measured -represented in the Hilbert space $\mathcal{H}_{S}$ and with Hamiltonian $H_{S}$ - and the measuring device $D$-represented in the Hilbert space $\mathcal{H}_{D}$ and with Hamiltonian $H_{D}$ - do not interact. During the second stage, an interaction Hamiltonian $H_{S D}^{\text {int }}$ introduces the correlation between the eigenstates $\left|o_{i}\right\rangle$ of the observable $O$ of $S$ and the eigenstates $\left|p_{i}\right\rangle$ of the pointer $P$ of $D$ (see Mittelstaedt 1998 for the precise form of $H_{S D}^{\text {int }}$ ). In the third stage the interaction ends, and the whole system becomes a composite system $S+D$ with a Hamiltonian $H=H_{S} \otimes I_{D}+I_{S} \otimes H_{D}$ and an initial state $\left|\psi_{S D}\right\rangle=\sum c_{i}\left|o_{i}\right\rangle \otimes\left|p_{i}\right\rangle$. Although $\left|\psi_{S D}\right\rangle$ is an entangled state, since there is no interaction between the subsystems $S$ and $D$, the actualization rule has to be applied to each one of them independently. In particular, when applied to $D$, the rule states that the definite-valued observables are the Hamiltonian $H_{D}$ and all the observables commuting with $H_{D}$ and having, at least, the same degeneracy as $H_{D}$.

On the basis of the above description, according to HMI two conditions define a quantum measurement:

(a) During a period $\Delta t, S$ and $D$ must interact through an interaction Hamiltonian $H_{S D}^{\text {int }} \neq 0$ intended to introduce a correlation between the observable $O$ of $S$ and the pointer $P$ of $D$. The requirement of perfect correlation is not included as a defining condition of measurement, because the actualization rule explains the definite reading of the pointer $P$ even in non-ideal measurements, that is, when the correlation is not perfect. In this case, the rule also accounts for 
the difference between reliable and non-reliable measurements (Lombardi and Castagnino 2008, Section 6).

(b) The measuring apparatus $D$ has to be constructed in such a way that its pointer $P$ (i) has macroscopically distinguishable eigenvalues, and (ii) commutes with the Hamiltonian $H_{D}$ and has, at least, the same degeneracy as $H_{D}$.

This account of the quantum measurement has been used to explain how the initial -pure or mixed- state is reconstructed through measurement both in the ideal and in the non-ideal case, and has been successfully applied to the paradigmatic example of the Stern-Gerlach experiment, with perfect and non-perfect correlation, and also in the case of an imperfect collimation of the incoming beam (Lombardi and Castagnino 2008). However, this account seems to be at odds with the explanation given by the EID program, according to which the decoherence of the measuring apparatus in interaction with its environment is what causes the apparent "collapse" that suppresses superpositions. In fact, in the MHI, the environment is absent: after the interaction $D$ is a closed quantum system unitarily evolving with its own Hamiltonian $H_{D}$. Moreover, this seems to flagrantly contradict the fact that real measuring apparatuses are never isolated, but they interact significantly with their environments. In the following sections we shall show that the conflict is only apparent.

\section{The MHI Preferred Context}

The first step towards dissolving the conflict between MHI and EID is to understand that, in the account given by MHI, the measuring device $D$ is not the macroscopic apparatus $A$ designed by the experimentalist for measurement (eventually surrounded by a "bath" $B$ of particles in interaction with it), but the entire quantum system that interacts with the system $S$ in the second stage and remains closed in the third stage: it is this system what must have a pointer commuting with its Hamiltonian $H_{D}$. On this basis, we can now analyze the elements that participate in the process as described in the framework of the MHI:

- The closed system $D$-e.g., the apparatus $A$ plus the bath of particles $B$ - is certainly a macroscopic system, whose Hamiltonian is the result of the interaction among a huge number of degrees of freedom. Since, in general, symmetries are broken by interactions, the symmetry of a Hamiltonian decreases with the complexity of the system. Then, a macroscopic system having a Hamiltonian with symmetries is a highly exceptional situation: in the generic case, the energy is the only constant of motion of the macroscopic system. As a consequence, in realistic measurement situations, $H_{D}$ is non-degenerate, 


$$
H_{D}\left|\omega_{n}\right\rangle=\omega_{n}\left|\omega_{n}\right\rangle \quad \text { with } \omega_{n} \neq \omega_{n^{\prime}}
$$

and, therefore, $\left\{\left|\omega_{n}\right\rangle\right\}$ is a basis of the Hilbert space $\mathcal{H}_{D}$ of $D$. This means that, when $\left[P, H_{D}\right]=0$, we can guarantee that $P$ has, at least, the same degeneracies as $H_{D}$ because $H_{D}$ is non-degenerate.

- The pointer $P$ cannot have such a huge number of different eigenvalues as $H_{D}$, because the experimental physicist must be able to discriminate among them (for instance, in the SternGerlach experiment the pointer has three eigenvalues). This means that $P$ is a "collective" observable of $D$ (see Omnés 1994, 1999), that is, a highly degenerate observable that does not "see" the vast majority of the degrees of freedom of $D$ :

$$
P=\sum_{n} p_{n} P_{n}
$$

where the set $\left\{P_{n}\right\}$ of the eigenprojectors of $P$ spans the Hilbert space $\mathcal{H}_{D}$ of $D$. In other words, the eigenprojectors of $P$ introduce a sort of "coarse-graining" onto the Hilbert space $\mathcal{H}_{D}$. Therefore, if the Hamiltonian $\mathcal{H}_{D}$ is non-degenerate (see eq.(7)), the condition $\left[P, H_{D}\right]=0$ implies that $P$ can be expressed in terms of the energy eigenbasis $\left\{\left|\omega_{n}\right\rangle\right\}$ as

$$
P=\sum_{n} p_{n} P_{n}=\sum_{n} p_{n} \sum_{i_{n}}\left|\omega_{i_{n}}\right\rangle\left\langle\omega_{i_{n}}\right|
$$

This expression shows that, since $p_{n} \neq p_{n^{\prime}}, P$ has more degeneracies than $H_{D}$.

- The requirement $\left[P, H_{D}\right]=0$, far from being an ad hoc condition necessary to apply the actualization rule, has a clear physical meaning: it is essential to preserve the stationary behavior of $P$ during the third stage of the measurement process, in order to make the reading of $P$ possible. If this requirement did not hold because of the uncontrollable interaction among the microscopic degrees of freedom of the macroscopic apparatus or between the macroscopic apparatus and an external "bath", the reading of $P$ would constantly change and measurement would be impossible. Therefore, the complete experimental arrangement has to be designed in such a way that the uncontrollable degrees of freedom of $D$-internal or external to the macroscopic apparatus- do not affect significantly the stationarity of the pointer. This goal may be achieved by many different technological means; but, in any case, measurement has to be a controlled situation where the reading of a stable pointer can be obtained.

\section{The EID Pointer Basis from a Closed System Perspective}

In the context of EID, during the third stage the measuring apparatus $M$ does no longer interact with the measured system $S$ but interacts with the environment $E$. If, in the context of MHI, we use 
$D=M+E$ to call the whole system that interacts with $S$ in the second stage but remains closed during the third stage, the question is how to identify the open parts of $D$ to be conceived as the measuring apparatus $M$ and the environment $E$. This is a legitimate question because a whole closed system may be partitioned in many different ways, none of them more "essential" than the others (Harshman and Wickramasekara 2007a, b; for this claim in the context of decoherence, see Castagnino, Laura and Lombardi 2007).

A natural assumption is to consider the macroscopic, material apparatus $A$ built for measurement as "the measuring apparatus" $M$, and the bath $B$ of the particles scattering off $A$ as "the environment" $E$; then, $D=A+B$ is the closed system resulting from the interaction between $A$ and $B$. From this position, it is supposed that $A$ is the open system that decoheres: the reduced density operator $\rho_{r}^{A}(t)$ of $A$ should converge to a final time-independent $\rho_{r}^{A}$, diagonal in the pointer basis of $A$, that is, of its Hilbert space $\mathcal{H}_{A}$, and the pointer $P$ should define such a basis. However, although apparently "natural", this is not the best choice for the split of $D$, since it does not take into account the environment internal to the device $A$. In fact, being a macroscopic body, $A$ has a huge number of degrees of freedom, which have to be "coarse-grained" by $P$ if it is to play the role of the pointer. In other words, since the pointer $P$ must have a small number of different eigenvalues to allow the observer to discriminate among them, $P$ is a highly degenerate observable on the Hilbert space $\mathcal{H}_{A}$ of the open macroscopic apparatus $A$ and, as a consequence, it does not define a basis of $\mathcal{H}_{A}$.

Since a closed quantum system can be partitioned in many, equally legitimate manners, $D$ can be split in a theoretically better founded way in the measurement case. Let us recall that the pointer $P$ is the observable whose eigenvectors became correlated with the eigenvectors of an observable of the measured system during the second stage of the process, and that the interaction in that stage was deliberately designed to introduce such a correlation. So, if we want that during the third stage $P$ really defines a basis, the open "measuring apparatus" $M$ must be the part of $D$ corresponding to the Hilbert space $\mathcal{H}_{M}$ where the pointer is non-degenerate. If we call $P_{M}$ the pointer belonging to $\mathcal{H}_{M} \otimes \mathcal{H}_{M}$, it reads

$$
P_{M}=\sum_{i} p_{n}\left|p_{n}\right\rangle\left\langle p_{n}\right|
$$

where $\left\{\left|p_{n}\right\rangle\right\}$ is a basis of $\mathcal{H}_{M}$. Then, the relevant partition is $\mathcal{H}_{D}=\mathcal{H}_{M} \otimes \mathcal{H}_{E}$, where $\mathcal{H}_{E}$ is the Hilbert space of the "environment" $E$, with basis $\left\{\left|e_{m}\right\rangle\right\}$. Then, the pointer acting on $\mathcal{H}_{D}$ can be expressed as a highly degenerate observable: 


$$
\begin{aligned}
& P=P_{M} \otimes I_{E}=\left(\sum_{n} p_{n}\left|p_{n}\right\rangle\left\langle p_{n}\right|\right) \otimes\left(\sum_{m}\left|e_{m}\right\rangle\left\langle e_{m}\right|\right)= \\
& =\sum_{n} p_{n} \sum_{m}\left|p_{n}\right\rangle \otimes\left|e_{m}\right\rangle\left\langle p_{n}\right| \otimes\left\langle e_{m}\right|=\sum_{n} p_{n} P_{n}
\end{aligned}
$$

This agrees with the features of $P$ required by MHI: $P$ introduces a sort of "coarse-graining" onto the Hilbert space $\mathcal{H}_{D}$ (compare eq.(11) with eq.(8)). The many degrees of freedom corresponding to the degeneracies of $P$ in $\mathcal{H}_{D}$ play the role of the "environment" $E$, composed by the microscopic degrees of freedom of the macroscopic apparatus $A$-internal environment- and the degrees of freedom of the bath $B$-external environment-.

\section{Compatibility between MHI and EID}

As we have seen, in the first papers on decoherence, the condition $\left[P, H_{M E}^{\mathrm{int}}\right]=0$ was considered as the definition of the pointer basis. However, this definition involves several assumptions. In fact, the entangled state $\left|\psi_{S M E}(t)\right\rangle \mid$ of the whole system evolves according to the Schrödinger equation under the action of the total Hamiltonian $H_{S M E}=H_{S}+H_{M}+H_{E}+H_{S M}^{\mathrm{int}}+H_{S E}^{\mathrm{int}}+H_{M E}^{\mathrm{int}}$. So, first it is considered that the system-environment interaction and the system-apparatus interaction are zero: $H_{S E}^{\mathrm{int}}=0$ and $H_{S M}^{\mathrm{int}}=0$. This assumption is reasonable on the basis of the design of the measurement arrangement: after a short time, any interaction with the system ends and the subsystem $M+E$ follows its independent dynamical evolution; for this reason, also the self-Hamiltonian $H_{S}$ of the system can be disregarded. Then, the stability of the pointer strictly requires that:

$$
\left[P, H_{M E}\right]=0 \quad \text { with } \quad H_{M E}=H_{M} \otimes I_{E}+I_{M} \otimes H_{E}+H_{M E}^{\mathrm{int}}
$$

If we recall that the pointer $P$ is an observable highly degenerate in the -internal and externaldegrees of freedom of the environment (see eq.(11)), then condition (12) results

$$
\left[P, H_{M E}\right]=\left[P_{M} \otimes I_{E}, H_{M} \otimes I_{E}+I_{M} \otimes H_{E}+H_{M E}^{\mathrm{int}}\right]=0
$$

But since $\left[P_{M} \otimes I_{E}, I_{M} \otimes H_{E}\right]=0$, then the stability requirement for the pointer observable becomes that it commutes with the Hamiltonian $H_{M} \otimes I_{E}+H_{M E}^{\text {int }}$, where the self-Hamiltonian of the environment is not involved:

$$
\left[P, H_{M} \otimes I_{E}+H_{M E}^{\mathrm{int}}\right]=0
$$

This argument shows that the condition $\left[P, H_{M E}^{\mathrm{int}}\right]=0$, introduced in the first papers on decoherence, is a particular case that holds only when the self-Hamiltonian $H_{M}$ of $M$ can be disregarded. It is also clear that the three regimes distinguished by Zurek as the result of the 
application of the predictability sieve turn out to be the three particular cases of condition (14), and can be redescribed in terms of that condition:

When $H_{M} \otimes I_{E}<H_{M E}^{\mathrm{int}}$, the self-Hamiltonian of $M$ can be neglected, and then $\left[P, H_{M E}^{\mathrm{int}}\right]=0$. Therefore, the preferred basis is defined by the interaction Hamiltonian $H_{M E}^{\text {int }}$.

$>$ When $H_{M} \otimes I_{E} \simeq H_{M E}^{\text {int }}$, neither the self-Hamiltonian of $M$ nor the interaction with the environment are clearly dominant. In this case, the preferred basis is defined by condition (14).

When $H_{M} \otimes I_{E}>H_{M E}^{\mathrm{int}}$, the dynamics is dominated by the self-Hamiltonian of $M$ and, then, $\left[P, H_{M} \otimes I_{E}\right]=\left[P_{M} \otimes I_{E}, H_{M} \otimes I_{E}\right]=\left[P_{M}, H_{M}\right]=0$. Therefore, the preferred states are simply the eigenstates of $H_{M}$.

As a consequence, the fact (noted by Schlosshauer 2004, 1280; see also Schlosshauer 2007, 84-85) that many systems are typically found in energy eigenstates although the interaction Hamiltonian depends on an observable different than energy, far from being surprising, necessarily results from the requirement of stability for the preferred basis. But the point we want to stress here is that, when the EID pointer basis is considered from this closed-system viewpoint, it agrees with the preferred context as defined by the MHI actualization rule: in both cases, the pointer/preferred basis is given by the Hamiltonian of the whole closed system. In fact, the three regimes identified and obtained case by case by Zurek turn out to be particular cases of the MHI characterization of the preferred basis: if the preferred states are defined by the eigenstates of the Hamiltonian of the whole system, it is not hard to realize that they will depend on the Hamiltonian's component which dominates the whole evolution.

Moreover, from this perspective the first regime can be justified on general grounds. According to Zurek, the first regime is the quantum measurement situation, where the selfHamiltonian of the measuring system $M$ can be neglected and the evolution is completely dominated by the interaction Hamiltonian: this means that $H_{M} \otimes I_{E}<<H_{M E}^{\mathrm{int}}$. If, as explained in the previous section, $M$ is the part of the closed system $D$ "viewed" by the pointer $P$ and the environment carries over almost all the degrees of freedom of $D$, it seems reasonable to suppose that, in general, the Hamiltonian corresponding to the interaction with that huge number of degrees of freedom is much greater than the self-Hamiltonian of the "small" part defined by the pointer: the condition $H_{M} \otimes I_{E}<<H_{M E}^{\text {int }}$ leading to the first regime turns out to have a physical justification. 


\section{Conclusions}

At present it is quite clear that the theory of decoherence does not supply an interpretation of quantum mechanics. Nevertheless, given its impressive success, it is also clear that nowadays no interpretation can ignore the results coming from the EID approach. The MHI has proved to be effective for solving the measurement problem with no need of EID. However, since the actualization rule applies to closed systems, the MHI seems to stand at odds of EID.

In this paper we have shown that this assumption is misguided. On the contrary, when the measurement process is viewed from a closed-system perspective, the MHI and the EID accounts of measurement agree: the classical-like states einselected by the interaction with the environment (the eigenvectors of the pointer, elements of the pointer basis) are the eigenvectors of an actual-valued observable belonging to the preferred context selected by the MHI.

\section{References}

Albert, David. 1992. Quantum Mechanics and Experience. Cambridge: Harvard University Press.

Albert, David, and Barry Loewer. 1990. "Wanted Dead or Alive: Two Attempts to Solve Schrödinger's Paradox." In Proceedings of the 1990 Biennial Meeting of the Philosophy of Science Association, Vol. 1, 277-85. East Lansing: Philosophy of Science Association.

-_-. 1993. "Non-Ideal Measurements." Foundations of Physics Letters 6: 297-305.

Ardenghi, Juan Sebastián, Mario Castagnino, and Olimpia Lombardi. 2009. "Quantum Mechanics: Modal Interpretation and Galilean Transformations." Foundations of Physics 39: 1023-45.

Bacciagaluppi, Guido. 2000. "Delocalized Properties in the Modal Interpretation of a Continuous Model of Decoherence." Foundations of Physics 30: 1431-44.

Bacciagaluppi, Guido, and Meir Hemmo. 1996. "Modal Interpretations, Decoherence and Measurements." Studies in History and Philosophy of Modern Physics 27: 239-77.

Castagnino, Mario, Roberto Laura, and Olimpia Lombardi. 2007. "A General Conceptual Framework for Decoherence in Closed and Open Systems." Philosophy of Science 74: 968-80.

Castagnino, Mario, and Olimpia Lombardi. 2008. "The Role of the Hamiltonian in the Interpretation of Quantum Mechanics.” Journal of Physics. Conferences Series 28: 012014. 
Dieks, Dennis. 1988. "The Formalism of Quantum Theory: An Objective Description of Reality?." Annalen der Physik 7: 174-90.

Dieks, Dennis. 1994a. “Objectification, Measurement and Classical Limit According to the Modal Interpretation of Quantum Mechanics." In Proceedings of the Symposium on the Foundations of Modern Physics, ed. Paul Busch, Pekka Lathi, and Peter Mittelstaedt, 160-67. Singapore: World Scientific.

Dieks, Dennis. 1994b. "The Modal Interpretation of Quantum Mechanics, Measurement and Macroscopic Behavior." Physical Review D 49, 2290-2300.

Dieks, Dennis, and Pieter Vermaas. 1998. The Modal Interpretation of Quantum Mechanics. Dordrecht: Kluwer Academic Publishers.

Elby, Andrew. 1993. "Why «Modal» Interpretations Don't Solve the Measurement Problem." Foundations of Physics Letters 6: 5-19.

Elby, Andrew. 1994. “The 'Decoherence' Approach to the Measurement Problem in Quantum Mechanics." In Proceedings of the Philosophy of Science Association, 1: S355-S365. East Lansing: Philosophy of Science Association.

Harshman, Nathan, and Sujeeva Wickramasekara. 2007a. "Galilean and Dynamical Invariance of Entanglement in Particle Scattering." Physical Review Letters 98: 080406.

-_-. 2007b. "Tensor Product Structures, Entanglement, and Particle Scattering." Open Systems and Information Dynamics 14: 341-51.

Healey, Richard. 1989. The Philosophy of Quantum Mechanics: An Interactive Interpretation. Cambridge: Cambridge University Press.

Healey, Richard. 1995. "Dissipating the Quantum Measurement Problem.” Topoi 14: 55-65.

Kochen, Simon. 1985. "A New Interpretation of Quantum Mechanics." In Symposium on the Foundations of Modern Physics, ed. Pekka Lahti, and Peter Mittelsteadt, 151-69. Singapore: World Scientific.

Lombardi, Olimpia, and Mario Castagnino. 2008. "A Modal-Hamiltonian Interpretation of Quantum Mechanics." Studies in History and Philosophy of Modern Physics 39: 380-443.

Lombardi, Olimpia, Mario Castagnino, and Juan Sebastián Ardenghi. 2010. "The ModalHamiltonian Interpretation and the Galilean Covariance of Quantum Mechanics." Studies in History and Philosophy of Modern Physics forthcoming. 
Mittelstaedt, Peter. 1998. The Interpretation of Quantum Mechanics and the Measurement Process. Cambridge: Cambridge University Press.

Monton, Bradley. 1999. "Van Fraassen and Ruetsche on Preparation and Measurement." Philosophy of Science 66, S82-S91.

Omnès, Roland. 1994. The Interpretation of Quantum Mechanics. Princeton: Princeton University Press.

---. 1999. Understanding Quantum Mechanics. Princeton: Princeton University Press.

Paz, Juan Pablo. 1994. "Decoherence in Quantum Brownian Motion." In Physical Origins of Time Asymmetry, ed. Jonathan Halliwell, Juan Pérez-Mercader, and Wojciech Zurek, 213-20. Cambridge: Cambridge University Press.

Paz, Juan Pablo, and Wojciech Zurek. 1999. "Quantum Limit of Decoherence: Environment Induced Superselection of Energy Eigenstates." Physical Review Letters 82: 5181-85.

--_. 2002. "Environment-Induced Decoherence and the Transition from Quantum to Classical." Fundamentals of Quantum Information, Lecture Notes in Physics, Vol. 587. ed. Dieter Heiss. Heidelberg-Berlin: Springer-Verlag. Also in arXiv:quant-ph/0010011.

Ruetsche, Laura. 1996. "Van Fraassen on Preparation and Measurement." Philosophy of Science 63, S338-S346.

Schlosshauer, Maximilian. 2004. "Decoherence, the Measurement Problem, and Interpretations of Quantum Mechanics." Reviews of Modern Physics 76: 1267-1305.

---. 2007. Decoherence and the Quantum-to-Classical Transition. Heidelberg-Berlin: Springer.

van Fraassen, Bas. 1991. Quantum Mechanics: An Empiricist View. Oxford: Oxford University Press.

Vermaas, Pieter, and Dennis Dieks. 1995. "The Modal Interpretation of Quantum Mechanics and Its Generalization to Density Operators." Foundations of Physics 25: 145-58.

Zurek, Wojciech. 1981. "Pointer Basis of Quantum Apparatus: Into What Mixtures does the Wave Packet Collapse?." Physical Review D 24: 1516-25.

---. 1982. "Environment-Induced Superselection Rules." Physical Review D 26:1862-80.

---. 1993. "Preferred States, Predictability, Classicality and the Environment-Induced Decoherence." Progress of Theoretical Physics 89: 281-312. 
---. 2003. "Decoherence, Einselection, and the Quantum Origins of the Classical," Reviews of Modern Physics 75: 715-76.

Zurek, Wojciech, Salman Habib, and Juan Pablo Paz. 1993. "Coherent States Via Decoherence." Physical Review Letters 70: 1187-90. 